\title{
THE AESTHETIC VALUE TO REHEARSE THE REALIZED FORMATIVE SHAPE AND ITS ROLE TO ACHIEVE THE COGNITIVE AND FUNCTIONAL COMPLEMENTARY THE PRINTED CHILD'S REQUIREMENTS
}

\author{
Noha Mahmoud Mohamed Ali \\ Assistant Professor, Alexandria University, Egypt, mohamedd@wtc-egypt.com
}

\begin{abstract}
The state has a great interest of the child arts, requirements and needs as the child is the main base to grow the next generation and to develop the human resources, it's an axial of in the development operation in the all societies so, it should be studied from the realized formative conceptions and symbolic and aesthetic characteristic which are expressive about the visual experience as variables and various subjects to inspire the artist and the graphic designer the printed works to reach to the values and aesthetic conceptions collaborate between the drawings, the functional causal performance to aim to fulfil the various requirements of the child and they are from the insight character of the child.

The modern studies are proved that there creative and artistic abilities to the child in different nationalities and environments, so the difference attributes to the degree of these abilities and not their kinds and the continue of them in the children depend on the discovery of them and assist the child to wide and give it a chance to save the various artistic values to appear these creative abilities according to the ages segments. So can we activate the artistic values in the realized formative output in the children's drawings to enrich their printed requirements? What are the aesthetic values of the realized formative output to enhance the self-learning skills to achieve the complementary cognitive? And what is the possibility to benefit from the Aesthetic values to the formative output to achieve the complementary between the functional and cognitive sides for the printed child's requirements? The research aims to benefit from the visual experience of the child which appear in the artistic symbols and drawings in all the formative values, creative visions, expressive contents and self-attitudes on the difference of realized formative items and rehearsing them in works or products can fulfil the printed child's requirements whereas the cognitive side is completed in different things which are presented in different characteristics in different fields of science in the education and the functional sides in different kinds to fulfill the benefit in different fields of daily life for the child from some wall- paintings and some disguising clothes which are enriched the creativity soul of the child, increase the power of positive motive and the artistic skills in the printed self-learning forever through finding the problems solutions to stimulate the forever motive of the child's creativity which sustain the self-confidence of him and to change the self-theory in different situations of life
\end{abstract}

Keywords: Aesthetic values, Realized Formative shape, The functional and cognitive complementary 


\section{INTRODUCTION}

The state now depends on the good investment of future generations through education to achieve the development of human resources because it is the focus of the development process in all societies so it was necessary to search for new teaching entries linking the field of hand printing in various other fields of science to increase the child's effectiveness and indirect approach to access the skills of self-education Which makes the child the focus of the learning process and supports the role of positive and effective result in improving the quality of the outputs of the educational process and add innovative ideas for their work.

Each child constructs his or her own formality with all the structures, lines, bodies and gradations, and builds all the learning experiences and visual visions to help him connect all his learning and add meaningful concepts to it, developing his confidence in himself and his ability to experiment and practice artistic expression techniques And to build his knowledge in an indirect way, which makes him comfortable with what he learns and to enhance learning and create a spirit of motivation for achievement, including personal, environmental, social, collectivity, financial and other factors. Each child is aware of his / her expressive and aesthetic values. Knowledge, and life. The knowledge is integrated, acquired, re-employed, creative and employed in creative solutions and functional artistic products with multiple functionalities that suit the needs of the child and society.

The research aims to benefit from the child's visual experiences, which appear in his artistic symbols and drawings with all the values of the creative and creative visions expressive and self-tendencies on the difference of those vocabulary and formal formulations in products that meet the child's printed requirements where the knowledge is integrated with all the knowledge, characteristics and features And the various fields of science within the educational process on the one hand and the complementarity and functional aspects of different kinds to meet its utilitarian purposes in all fields of daily life of the child from the work of mural, pendants, costumes and disguise ... and other hand which The creative energies of the child increase his positive motivation and his skills in daily self-learning through finding solutions to the technical problems that face him. This raises constant motivation to solve them, which in turn stimulates his creative energies on an ongoing basis, thus reinforcing his self-confidence and changing his self-image.

\section{SEARCH PROBLEM BACKGROUN}

Recent studies have shown that there are creative artistic abilities of children of different nationality and environment. The difference in the presence of these abilities in children is due to their degree and not to their type. The continuation of their continuation depends on their discovery and helps the child to expand it. Age:

The research problem is determined by the following questions:

Can the aesthetic values of the formalized formulations in the child's drawings be activated to enrich the printed requirements ?What is the role of the aesthetic values of the formal mind in promoting and communicating the skills of self-learning in order to achieve cognitive integration? What is the potential to benefit from the aesthetic values of formal consciousness in the integration of the functional knowledge of the child's printed requirements What is the role of the aesthetic values of the formal mind in promoting and communicating the skills of self-learning in order to achieve cognitive integration? What is the potential to benefit from the aesthetic values of formal consciousness in the integration of the functional knowledge of the child's printed requirements?

\section{RESEARCH GOALS}

Studying the aesthetic values of the artistic connotations and the expressive symbolic dimensions of the formal form factor as a child.

Activating the aesthetic values of the formal consciousness as a formality to enrich the printed child's requirements.

Strengthening and continuing the skills of self-learning by taking advantage of the role of aesthetic values of the formal consciousness to achieve the integration of knowledge.

Confirmation of the aesthetic entries of aesthetic values in formative formulations in achieving the integration between the cognitive and functional aspects of the child's needs in all their utilitarian roles.

\section{RESEARCH IMPORTANCE}

Shedding light on the aesthetic concepts and artistic connotations and the expressive and symbolic trends of the formal formality as a vocabulary in the child. To highlight the importance of knowledge integration 
between the various fields of knowledge and the integration of experiences to stimulate and raise the child's motivation to continue learning in various fields .Emphasize the skills of self - learning and promotion of print and increase the child's effectiveness and self - confidence to continue experimentation. Assure the vision of the community and build the personality of the child integrated ideologically and skillfully and spiritually and prepare a generation capable of facing all the global variables. Rephrasing the formal perception as a form factor to enrich the child's print needs aesthetically and functionally opens up new horizons for linking the arts to the true requirements of society.

\section{RESEARCH METHODOLOGY}

The research follows the analytical descriptive method in the theoretical aspect and follows the semiempirical approach through application of applied research experience.

\section{EMOTIONAL AND SYMBOLIC DIMENSIONS OF THE CHILD'S FORMAL FORM}

The child understands the outside world around him by means of a special fee for each child through which he expresses people, elements and things, affirming their relationship with the inner world and the connection between them. He reiterates his own formal perceptions to achieve psychological enjoyment as he changes his drawings according to his emotions. Value during expression and deletion of insignificant parts, use of the earth line to express the connection of the outside world to the inner world, the sense of distant neighbor, expression of objects, perception of the meaning of color and its relation to the external environment and the use of specific colors of certain elements, Based on the intellectual and cognitive facts in the expression and the emergence of certain trends such as flatness and transparency with the combination of different surfaces and the combination of different times and places and semi-geometric lines in the drawings followed by attempts and transformation of the attempts of simultaneous real expression with the integrated stages of growth and starts a logical direction, The distinctive details of elements, the awareness of proportions, the relation of parts to the whole, the distinction of visual orientation based on visual values in all, and self-direction based on personal self-emotions.

\section{SEARCH EXPERIENCE}

The child employs the skills of self-learning at all stages of drawing, designing and implementing his work from the beginning of receiving information and knowledge in various fields of science inside and outside the educational institutions in cooperation with the teacher of art education from pre-start observation of the task and all information received and linked with previous knowledge, And the extent of his ability to support the teacher to connect with the current cognitive system and self-observation and reflection and in the process of sharing his opinion and follow each stage self-evaluation and self-esteem and try to benefit from everything provided by the surrounding environment And determines the goal, it observes during the visual vision and read and listen and employ senses and looking for details and then deduces and distinguishes and presents solutions to the problem of technical self-awareness and takes the decision and passes through these stages of the sense of enjoyment of activity and links the information of his personal nature and link the technical task of his own work and evidence to draw conclusions to link the current experiences of the previous And passes the subsequent experiences to achieve the integrated development of the child's personality in various aspects and then innovates and develops theories and hypotheses and invent creative solutions.

The focus of experimentation is the basis of artistic work where the child is applied discovers up energy, flexibility and originality in successful experiments to solve the technical his problem creative and connects the various fields together uses other techniques and different materials in the technical scope of work and achieve through so many aesthetic and expressive values overlapping areas of art education together works of art and photography and art printing barriers between Wiesel cognitive aspects are integrated in various fields and be integrated into his artistic work cognitive and executive Mharria which leaves its impact on the child's personality Allowajaddana acquires confidence in his own abilities and the abilities and skills $b$ To the tune of harmony, diversity, complexity and simplification of organic unity and artistic style of his work depends change their minds on the same Inquisition, criticism, organization and reorganization in different ways with self-orientation, skill and personal compatibility and responsibility by the society towards.

\subsection{Experiments}

The research experience was based on the presentation of the formal drawings of the child's drawings through the stages of artistic expression from the age of seven to the age of 18 through the various stages and their characteristics and various characteristics in terms of the intellectual content of the drawings and style and aspects of the subjects and the use of art as a language of continuity in all its types and its link to the growth stage A variety of children with commitment to the age stages of time and directing them to draw 
different topics and elements commensurate with the stages of their growth.

Each child was directed to the tools and raw materials to help him in his artistic expressions without interfering in his style and his way with increasing the child's experience and awareness of the center in which he lives in order to develop the child's awareness and diversity of symbols and many and attract his attention to other elements not known around him and create the appropriate environment to help him to continue the expression with enjoyment To become an intellectual imagination and connect between the symbols of the motor and called the imaginary visions of self-development and feel the entity and be completed expertise and awareness of the external environment in the same way in the expression and continue to practice the activity of art and emphasize his own experience in the selection of topics and And his mental and social aspects in the stages of the greatest temporal life. His drawings were characterized by the features and characteristics of the child characteristic of him alone, along with the spontaneous freedom and exaggeration in the main functional parts of the subject and flattening and the elements alone according to knowledge and not the visual vision using the land line of vertical and horizontal situations carried out by the child Daily life activities, and with the overall growth of the age stages of the transition from the subjective direction of the subject and attention to the details of the distinctive formality and the disappearance of parts of elements and different colors according to the visual vision, so must be confirmed On the aesthetic relations in his artistic expressions by enriching self-awareness and individual differences and working in a team with the distinction of gender and the formation of two directions during the stages of integrated development from an objective visual orientation and an individual subjective direction in the full realization of the relations between things form and color. The optical direction emphasizes shade, light, In contrast to the self-direction which employs its emotions in symbols, color and size.

Thus, many different formative perceptions of children were obtained through the different age ranges, which are the starting points of the experiment that will be employed by the third year students in the Department of Art Education, Faculty of Specific Education, Alexandria University.

\subsection{Stages of the Application of the Experience}

The third year students to obtain variables and different perspectives of perceptions formal children reflect the different elements around them in society, the environment and the house has been converted their own artistic symbols express their ideas and their vision and their personality and art of those perceptions reflect everything around them cars and buildings and numbers arithmetic cuisine and trees animals and other reemployed with all contained characteristics and attributes and characteristics of the stages of development and growth in chronological age in each stage, which appear in the technical abilities and expressions of various shapes and elements that are specific to each child in patterns and frameworks symbols of the format becomes as b D artistic and expressive of semantics stand to appear in technical directions and symbols of plastic.

\subsection{Axes of Experience}

These perceptions were employed by the students based on two axes. The first is to employ formal perceptions from the plastic side to experimentally to adapt them aesthetically, with the aim of offering aesthetic and aesthetic solutions that enrich mental abilities, flexibility, fluency and originality, enhance unconventional thinking and self-learning skills, and increase the child's effectiveness, motivation and confidence. himself and that he is able to reach positive solutions and creative opportunity and unleash the creative imagination of the child and the second axis combining the aesthetic aspect and customized to fit the techniques of printing with all advanced color and visions of lbb interferences Conscious pigmentary and activate the above opportunist to perform the function required for the life of the child and the requirements of traditional costumes and murals and designs suitable for furniture inside the baby's room to benefit from the manual and technological capabilities for students to exit the final product.

\subsection{The Goal of the Experiment}

Re-employment of aesthetic values resulting from the planning of the formal perception resulting from different experiences visually and knowingly all the technical and expressive meanings and artistic trends included in their drawings and expressive contents in their formal symbols and confirm the role of cognitive integration of information, characteristics, characteristics, skills and various knowledge, skills and emotional and social about everything around them and distinctive features of each element and all the knowledge that is received educational and enriching Hiatia with self-learning skills and strengthen the part of the study in various sciences and promote the child's continued learning and education both in and accept the diversity of experiences and methods of receiving For the information and involvement of children in the printing and applied workshops in cooperation with the students of the third year increases the skills of self-learning 
printing and works on the continuity of life as a result of the search for permanent solutions to the technical problems that face it, which raises the motivation for permanent solution, which in turn stimulates creative energies permanently and persistently supports self-confidence and self-changing looks for all the positions of life and then invest the skills and aesthetics of printing technologies and supports enhanced self-learning skills typographic employ printing techniques Patek printing templates and stencils and dyeing techniques of contract Connecting with methods of folding and holding various dyes natural environment friendly safe to the child's safety experimentation with the versatility of useful recruitment of various requirements for the child (printing on the walls and walls and accessories Zinh of accessories and caps and bags and interfaces for pillows and brochures and children's room covers and blankets and belts and dolls and others to adapt these fees to meet the needs of the child to wear something from making his hand controls the colors and include the estimated value of manual labor and is proud of his design and build character and integrity and Tdamiha and thus achieve the integration of knowledge and receipt of acquisition and re-employment and creativity of its product and manifestation and ores Diverse techniques.

\subsection{Artworks of the Experiment}

-The need to develop contemporary strategies to promote the arts of the child to keep up with global thought and to create a generation capable of effective communication and acceptance of the other.

-Enhancing the cultural identity of the child and asserting it and linking the various sciences with culture and eliminating the gaps between the fields of knowledge and artistic education to achieve the integration of knowledge within the child's mind and enrich the mutual relations between science and the arts.

- Establishing a new generation capable of managing the integrated knowledge society and employing it in a positive and systematic role through continuous lifelong learning and outside the walls of educational institutions.

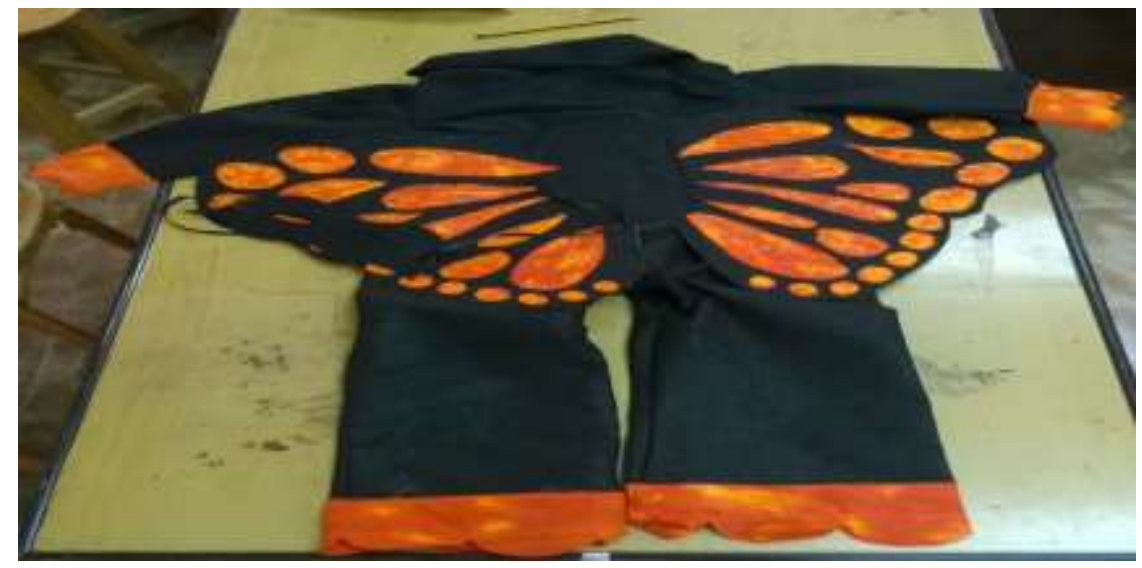

Fig. 1

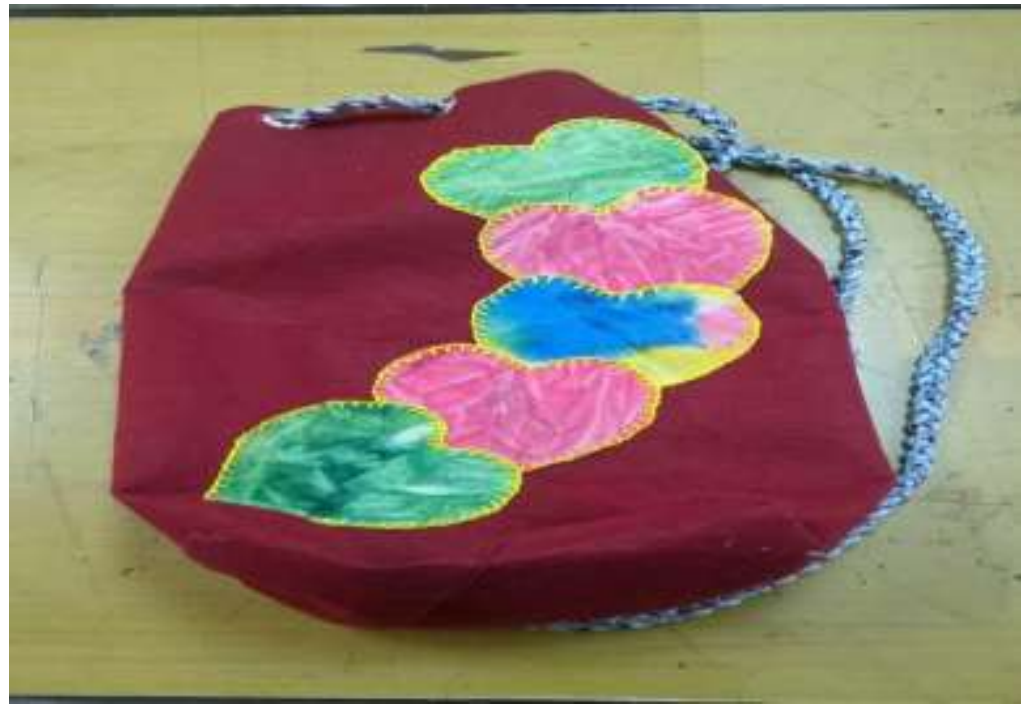

Fig. 2 


\section{RESULTS}

- The need to develop contemporary strategies to promote the arts of the child to keep up with global thought and to create a generation capable of effective communication and acceptance of the other.

- Enhancing the cultural identity of the child and asserting it and linking the various sciences with culture and eliminating the gaps between the fields of knowledge and artistic education to achieve the integration of knowledge within the child's mind and enrich the mutual relations between science and the arts.

- Establishing a new generation capable of managing the integrated knowledge society and employing it in a positive and systematic role through continuous lifelong learning and outside the walls of educational institutions.

\section{REFERENCES LIST}

Laila, Amr Abu and Nusseibeh Ahmed, without a date. "Your Talented Child", Dar Al Qalam, Algeria,

Ali Weddad Diab El Alaouna, 2015, A study of the stability of the evolutionary theory of the characteristics of the child in Victor and tradition of the age group (7-9 years), published research, research in the sciences and specific arts, Faculty of Specific Education, Alexandria University, vol 1, No. 3.

Adel, Dina Hassan, 2015, "Empowering Self-Learning Skills in Dynamic Museums to Experience Child Cognitive Integration in the Light of the Characteristics of the Knowledge Society", Research Paper, Research in Science and Specific Arts, Vol. I, No. 3.

Al-Jizani , Kazem, "Psychology of Children's Fees in Primary Schools", Volume of the News, Egypt, No. 72.

UNESCO World Report, From the Information Society to Knowledge Societies, United Nations Educational, Scientific and Cultural Organization, UNESCO Publications, Paris, 2005.

Hussein, Hamad Bin Al-Dowaihi, 2006, "The Role of Art and Artistic Education in Forming the Culture of the Arab Child", King Saud University Journal, Educational Sciences and Islamic Studies, Volume 18.

Thus, many different formative perceptions of children were obtained through the different age ranges, which are the starting points of the experiment that will be employed by the third year students in the Department of Art Education, Faculty of Specific Education, Alexandria University. 